(2) Open Access Full Text Article

\title{
Astragaloside IV represses high glucose-induced mesangial cells activation by enhancing autophagy via SIRTI deacetylation of NF-KB p65 subunit
}

This article was published in the following Dove Press journal: Drug Design, Development and Therapy

\author{
Xiaolei Wang ${ }^{1,2}$ \\ Yanbin Gao ${ }^{1,2}$ \\ Nianxiu Tian' \\ Zhiyao Zhu ${ }^{2}$ \\ Tao Wang' \\ Jiayi $\mathrm{Xu}^{\prime}$ \\ Bingjie $\mathrm{Wu}^{\prime}$ \\ Nan Zhang ${ }^{2}$
}

'Department of Endocrinology, School of Traditional Chinese Medicine, Capital Medical University, Beijing, China; ${ }^{2}$ Department of Endocrinology, Beijing Key Lab of Traditional Chinese Medicine Collateral Disease theory Research, Capital Medical University, Beijing, China
Correspondence: Yanbin Gao School of Traditional Chinese Medicine, Capital Medical University, No I0, Youanmenwai, Xitoutiao, Fengtai District, Beijing 100069, China

Tel +86 I0 839I I720

Email dfyynfm@।63.com
Aim: Mesangial cell (MC) activation plays an important role in many glomerular diseases associated with renal fibrosis, including diabetic kidney disease (DKD). The aim of this study was to determine whether Astragaloside IV (AS-IV) modulated MC activation in DKD via autophagy by specifically regulating the autophagy inducer sirtuin 1 (SIRT1).

Methods: Cultured MCs and diabetic KK-Ay mice were treated with AS-IV, and the markers and regulatory mediators of autophagy were analyzed using Western blotting, real-time PCR, ELISA and IF.

Results: AS-IV inhibited MC activation and enhanced autophagy in hyperglycemic conditions by increasing SIRT1 expression and decreasing NF- $\kappa B$ p 65 acetylation. In addition, the SIRT1 activator SRT1720 enhanced autophagy and decreased p65 acetylation during hyperglycemia-induced MC activation. Opposite effects were seen with the SIRT1 inhibitor EX527. Furthermore, the ameliorative effect of AS-IV on MCs was abolished by the autophagy inhibitor 3-MA, while the autophagy activator rapamycin restored hyperglycemia-induced MC activation. Finally, AS-IV improved renal function and fibrosis in the diabetic KK-Ay mice.

Conclusion: AS-IV ameliorated renal function and morphology by inducing autophagy and inhibiting MC activation through the SIRT1-NF- $\kappa$ B pathway, indicating a potential therapeutic role of AS-IV in glomerular diseases.

Keywords: Chinese medicine, pharmacological effect, diabetic kidney disease, SIRT1-NF-KB pathway, renal fibrosis

\section{Introduction}

Diabetic kidney disease (DKD), a microvascular complication of diabetes and the leading cause of chronic renal failure, is pathologically characterized as glomerulosclerosis. ${ }^{1}$ The pathological features of glomerulosclerosis are extracellular matrix (ECM) accumulation and mesangial cell (MC) proliferation. ${ }^{2} \mathrm{MCs}$ are activated when subjected to harmful stimuli, manifested as increased $\alpha$-smooth muscle actin ( $\alpha$-SMA) expression, and then proliferate and synthesize excessive amounts of ECM proteins. ${ }^{3}$ Therefore, it is necessary to explore the underlying mechanisms of MC activation in order to block the progression of diabetic glomerulosclerosis.

Sirtuin 1 (SIRT1), a deacetylase regulating several enzymes, proteins, and transcription factors, ${ }^{4,5}$ is involved in many physiological processes such as cellular senescence, ${ }^{6}$ metabolism, ${ }^{7}$ and stress responses. ${ }^{8}$ Studies have showed that SIRT1 plays a protective role in the onset and progression of DKD. ${ }^{7}$ A previous study showed that SIRT1 de-acetylated and inactivated NF- $\mathrm{KB}$ in MCs, thereby inhibiting their expansion and alleviating renal damage in a streptozotocin (STZ)-induced diabetic rat model. ${ }^{9}$ In 
addition, SIRT1 suppressed TGF- $\beta 1$-associated MC apoptosis via Smad7 deacetylation. ${ }^{10}$ Therefore, SIRT1 is a potential target for inhibiting MC injury in DKD.

Autophagy is an evolutionarily conserved process wherein cellular protein aggregates and damaged organelles are degraded to maintain intracellular homeostasis. It plays an important role in several kidney diseases including $\mathrm{DKD}^{11}$ and is also involved in MC injury. ${ }^{12-14} \mathrm{Xu}$ et $\mathrm{al}^{12}$ found that autophagy blocked advanced glycation end products (AGEs)-induced MC apoptosis by inhibiting the production of reactive oxygen species (ROS). Lu et $\mathrm{al}^{13}$ demonstrated that ursolic acid improved diabetic MC injury by enhancing autophagy. However, whether autophagy can also regulate MC activation and whether this mechanism is associated with the autophagy inducer SIRT1 is not fully understood.

Astragaloside IV (AS-IV), a bioactive saponin extracted from the Astragalus root, can exert a variety of pharmacological effects via its anti-oxidative and anti-inflammatory functions. ${ }^{15}$ Previous studies have shown that AS-IV attenuated MC injury induced by hyperglycemia by suppressing ROS production. ${ }^{16}$ Chen et $\mathrm{al}^{17}$ showed that AS-IV also inhibited hyperglycemia-induced MC proliferation. A recent study has shown that AS-IV protected against podocyte injury by enhancing autophagy in a STZ-induced diabetic mouse model. ${ }^{18}$ However, the effect of AS-IV on MC activation and the mediatory role of autophagy, if any, is unknown. Therefore, we investigated the effect of AS-IV in hyperglycemia-induced MC activation, with a major focus on the role of autophagy.

\section{Materials and methods}

\section{Reagents}

AS-IV (C41H68O14, molecular weight $=784.97$, purity by high-performance liquid chromatography [HPLC] $\geq 98 \%$ ) was purchased from Sigma-Aldrich Co. (St Louis, MO, USA). SRT1720 (S1129), EX527 (S1541), rapamycin (S1039), and 3-MA (S2767) were obtained from Selleck Chemicals (Houston, TX, USA). Rabbit polyclonal anti-alpha smooth muscle actin ( $\alpha$-SMA), anti-fibronectin (FN), anticollagen IV (Col IV), anti-LC3 II, anti-Beclin 1, anti-SIRT1,

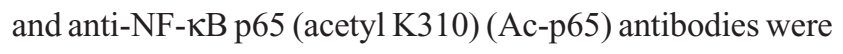
purchased from Abcam (Cambridge, UK).

\section{Cell culture}

The conditionally immortalized mouse glomerular MC line SV40 MES 13 was obtained from China Infrastructure of Cell Line Resources. The cells were cultured in low glucose (5.56 mM) Dulbecco's Modified Eagle's Medium (DMEM) supplemented with $10 \% \mathrm{FBS}, 100 \mathrm{U} / \mathrm{mL}$ penicillin, and
$100 \mu \mathrm{g} / \mathrm{mL}$ streptomycin under $5 \% \mathrm{CO}_{2}$ at $37^{\circ} \mathrm{C}$. For all experiments, cells were cultured in serum-free conditions for 24 hours when they reached $80 \%$ confluence.

\section{Establishment of DKD model and in vivo experimental design}

Eight-week-old male KK-Ay mice and male C57BL/6J mice (Chinese Academy of Medical Sciences, Beijing, China) were housed at constant room temperature $\left(24^{\circ} \mathrm{C}\right)$ and humidity (70\%) under a controlled light/dark cycle. All animals had access to water ad libitum. C57BL/6J mice were fed with standard diet (12\% fat, $28 \%$ protein and $60 \%$ carbohydrates). DKD was induced by feeding the KK-Ay mice with a high-fat diet (HFD; $58 \%$ fat, $16.4 \%$ protein and $25.6 \%$ carbohydrates) for 4 weeks. DKD was diagnosed when the random blood glucose was $\geq 16.7 \mathrm{mM}$ and urine albumin creatinine ratio (ACR) was $\geq 300 \mu \mathrm{g} / \mathrm{mg}$. The DKD mice were then randomized into the untreated (DKD group, $n=12$ ) and AS-IV treated (AS-IV group, $n=12$ ) groups. The untreated mice were given an oral gavage of aqua distillate, while the treated mice received oral gavages of AS-IV at $40 \mathrm{mg} / \mathrm{kg} /$ day. C57BL/6J mice were used as normal controls (NC group, $n=12$ ) and given aqua distillate gavage. After 12 weeks, blood and 24-hour urine were collected, and renal tissues were dissected. All experiments were approved by the Institutional Animal Care and Use Committee at Capital Medical University, conforming to the Guide for the Care and Use of Laboratory Animals by the National Institute of Health.

\section{Immunofluorescence (IF) staining}

The renal tissues were fixed in $4 \%$ paraformaldehyde, paraffin-embedded and sectioned for IF staining. The sections were deparaffinized, dehydrated, and boiled for antigen retrieval. After inhibiting endogenous peroxidase activity with $3 \%$ hydrogen peroxide, the sections were blocked with $5 \%$ goat serum for 30 minutes and then incubated overnight with the primary antibodies at $4{ }^{\circ} \mathrm{C}$. The following day, the sections were incubated with secondary antibody for 1 hour at $37^{\circ} \mathrm{C}$ and counterstained with DAPI. Stained cells were imaged with a fluorescence microscope. The primary antibodies used were the rabbit anti- $\alpha$-SMA (1:100), rabbit anti- $\alpha-$ SIRT1 (1:200), and rabbit anti-Ac-p65 (1:200).

\section{Real-time PCR analysis}

Total RNA of MCs was extracted using TRIzol reagent (Thermo Fisher Scientific, Waltham, MA, USA) according to the manufacturer's instructions. To detect the expression 
of nuclear p65 in MCs, the nuclei were first extracted with a nuclear fractionation kit (Solarbio, Beijing, China), followed by extraction of the nuclear RNA. Real-time PCR primers were designed as described previously. ${ }^{19}$ Relative expression was calculated using the comparative cycle threshold $(\mathrm{Ct})$ method $\left(2^{-\Delta \Delta} \mathrm{CT}\right) .{ }^{20}$ The relative expressions of $\alpha$-SMA, SIRT1, and p65 were normalized to GAPDH internal control. Three independent experiments were performed.

\section{Western blotting}

Total proteins from MCs and renal tissues were extracted, separated by SDS-PAGE and then transferred to PVDF membrane. The membranes were blocked with 5\% nonfat dry milk and then incubated overnight with primary antibodies at $4{ }^{\circ} \mathrm{C}$, followed by horseradish peroxidase-conjugated secondary antibody (1:1,000, Beyotime, Shanghai, China). The primary antibodies and dilutions used are as follows: rabbit polyclonal antibodies against $\alpha$-SMA $(1: 1,000)$, SIRT1 (1:1,000), Ac-p65 (1:400), LC3 II (1:3,000), and Beclin 1 $(1: 1,000)$. The specific bands were detected with enhanced chemiluminescence.

\section{Cell counting kit-8 (CCK-8) assay and enzyme-linked immunosorbent assay (ELISA)}

To detect MC proliferation, CCK-8 assay was performed according to the manufacturer's instructions. To determine ECM production, the supernatant of cultured MCs was collected and collagenase (Col) IV and fibronectin (FN) levels were quantified by ELISA according to the manufacturer's instructions (Blue Gene, Shanghai, China).

\section{Statistical analysis}

SPSS 19.0 software (IBM Corporation, Armonk, NY, USA) was used for statistical analysis. All data are presented as mean $\pm \mathrm{SD}$. Student's unpaired $t$-test was used to compare two groups and multiple groups were compared using one-way ANOVA. $P$-value $<0.05$ was considered statistically significant.

\section{Results}

\section{Effect of AS-IV on hyperglycemia-induced activation of MCs}

The proliferation rates of MCs exposed to high glucose conditions (30 $\mathrm{mM}$ ) for varying durations (24, 48, and 72 hours) were measured with the CCK-8 assay. A significant elevation in the proliferation rate was detected at 24 hours that peaked at 48 hours, and then declined after 72 hours of hyperglycemia (Figure 1A). The likely reason is that prolonged exposure to high glucose levels induced apoptosis in the MCs. To determine the effect of AS-IV on hyperglycemia-induced $\mathrm{MC}$ activation, the cells were pretreated with different concentrations of AS-IV $(25,50$, and $100 \mu \mathrm{M})$. AS-IV significantly decreased the proliferation rate of MCs after 48 hours of hyperglycemic exposure (Figure 1B). Furthermore, AS-IV pretreatment significantly lowered the levels of $\alpha$-SMA mRNA and protein (Figure 1C and D), as well as the FN and Col IV levels in hyperglycemic MCs in a dose-dependent manner (Figure $1 \mathrm{E}$ and $\mathrm{F}$ ). In addition, in situ level of $\alpha$-SMA was significantly higher in the glomeruli of the untreated DKD mice compared to that of NC mice, and lower compared to the AS-IV treated mice (Figure 1G). These results strongly suggested that hyperglycemia activated the MCs, which was restored by AS-IV in a dose-dependent manner.

\section{Effect of AS-IV on SIRTI expression in hyperglycemia-induced $M C$ activation and the role of NF- $\kappa B$}

To investigate the effect of AS-IV on SIRT1 expression in hyperglycemia-activated MCs, cells with/without AS-IV pretreatment were stimulated under high glucose conditions for 48 hours. SIRT1 expression was significantly decreased in the untreated MCs and restored by AS-IV (Figure 2A and B). To further validate the restorative effect of AS-IV on hyperglycemia-induced SIRT1 inhibition in MCs, the cells were treated with either SIRT1 activator SRT1720 or the inhibitor EX527 for 1 hour after high glucose exposure, and then incubated in the presence (or absence) of AS-IV for 48 hours. AS-IV significantly increased SIRT1 expression in MCs co-treated with SRT1720, while prior exposure to EX527 significantly decreased SIRT1 levels in AS-IVtreated MCs (Figure 2A and B). Taken together, these findings indicate that AS-IV mitigated SIRT1 inhibition during hyperglycemia-induced MC activation.

To determine whether AS-IV regulated SIRT1 via the NF- $\kappa B$ pathway, the expression of the NF- $\mathrm{kB}$ p 65 subunit was analyzed in MCs exposed to hyperglycemic conditions. High glucose levels increased the level of p65 acetylation, which was decreased upon AS-IV treatment (Figure 2C and D). Furthermore, SRT1720 significantly decreased and EX527 increased AC-p65 levels in MCs treated with AS-IV (Figure 2C and D). Taken together, our results indicate that AS-IV exerts its effect on hyperglycemia-activated MCs via the NF- $\mathrm{kB}$ pathway by SIRT1-mediated de-acetylation of p65 subunit.

We also detected the in situ expression of SIRT1 and AC-p65 in the mice glomeruli. SIRT1 expression was decreased and AC-p65 was increased in the glomeruli of 
A
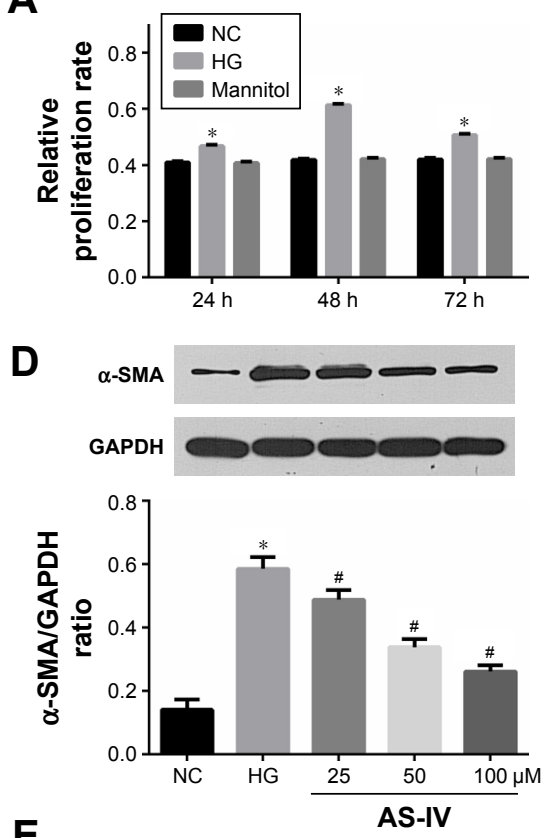

E
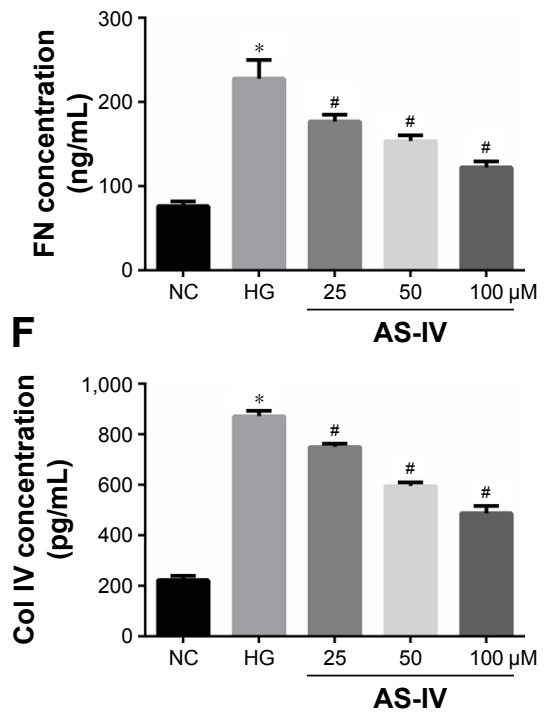

B

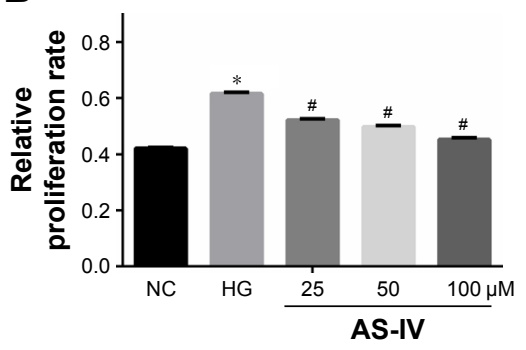

C

G

$\alpha$-SMA
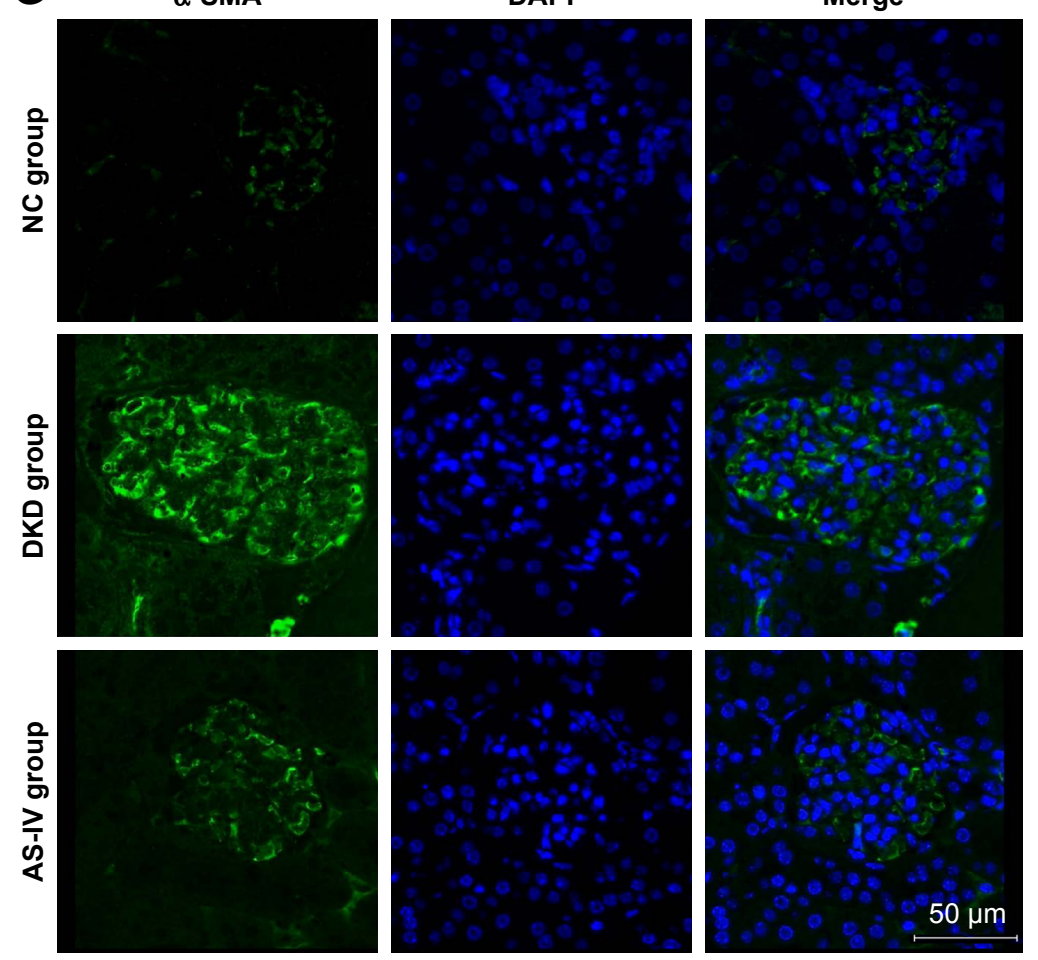

Figure I Effect of AS-IV on hyperglycemia-induced activation of MCs.

Notes: (A and B) CCK-8 assay results showed hyperglycemia increased the proliferation rate of MCs and that AS-IV decreased the proliferation rate of MCs treated with hyperglycemia for 48 hours in a dose-dependent manner. (C and D) Real-time PCR and Western blotting results showed AS-IV decreased the relative $\alpha$-SMA levels in MCs exposed to hyperglycemia for 48 hours in a dose-dependent manner. (E and F) ELISA assay results showed AS-IV decreased the relative FN and Col IV levels in hyperglycemia-stimulated MCs for 48 hours in a dose-dependent manner. (G) Immunofluorescence images showed that the expression of $\alpha$-SMA was increased in DKD group mice glomerulus compared with NC group mice glomerulus and that $\alpha$-SMA expression was decreased in AS-IV group mice glomerulus. Blue, nuclear staining (DAPI); Green, target protein staining. Data are presented as mean \pm SD. $n=3$. *Compared with NC, $P<0.05$; ${ }^{*}$ Compared with $\mathrm{HG}, P<0.05$.

Abbreviations: AS-IV, Astragaloside IV; CCK-8, cell counting kit-8; DKD, diabetic kidney disease; HG, high glucose; MC, mesangial cell; NC, normal control.

untreated DKD mice compared to the NC group, while AS-IV treatment increased SIRT1 expression and decreased AC-p65 expression (Figure 3).

\section{AS-IV and SIRTI-induced inactivation of NF-KB signaling results in autophagy in hyperglycemia-activated MCs}

A previous study reported that $\mathrm{NF}-\kappa \mathrm{B}$ signaling regulated autophagy via SIRT1 deacetylation. ${ }^{21}$ To explore the role of SIRT1-induced inactivation of NF- $\mathrm{KB}$ signaling in $\mathrm{MC}$ autophagy under hyperglycemic conditions, we analyzed the expression of the autophagy markers Beclin 1 and LC3 II. Hyperglycemia decreased the level of Beclin 1 in MCs, which was restored by AS-IV treatment (Figure 4A). LC3 II levels also increased significantly I the MCs after AS-IV treatment under hyperglycemic conditions (Figure 4B).

To further affirm the role of the SIRT1-NF- $\kappa$ B axis in regulating autophagy, MCs were treated with SRT1720 or EX527 

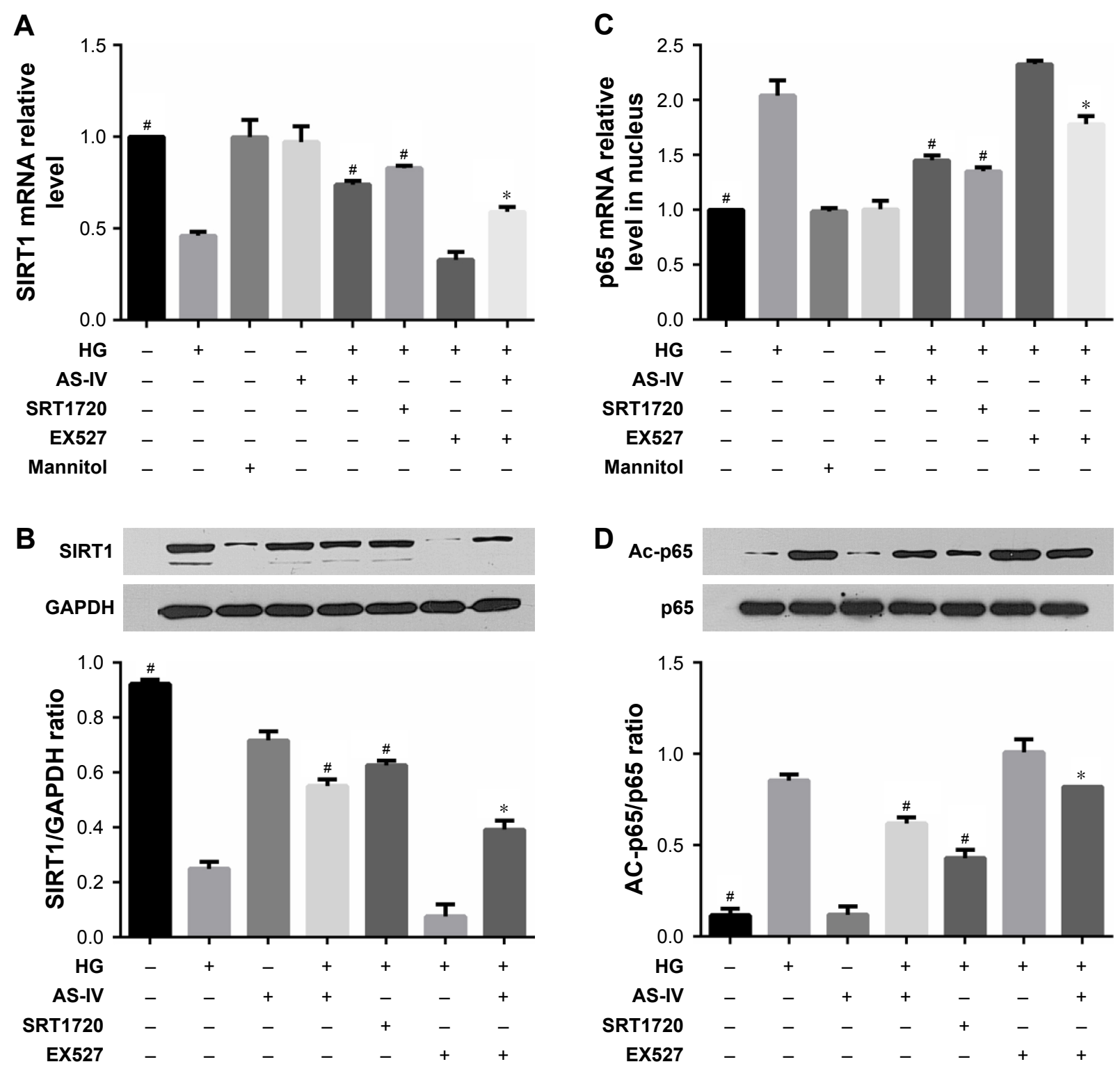

Figure 2 Effect of AS-IV on SIRTI and NF-KB p65 expression in hyperglycemia-induced MC activation in vitro.

Notes: (A and B) Real-time PCR and Western blotting results showed that both SIRTI activator SRTI720 and AS-IV increased the relative SIRTI level in MCs exposed to hyperglycemia for 48 hours and that SIRTI inhibitor EX527 abolished the change of AS-IV. (C and D) Real-time PCR and Western blotting results showed both SRTI720 and AS-IV decreased the relative 655 acetylation levels in MCs exposed to hyperglycemia for 48 hours and that EX527 increased the p65 acetylation levels. Data are presented as mean \pm SD. $n=3$. *Compared with co-treated with hyperglycemia and AS-IV group, $P<0.05$; ${ }^{\#}$ Compared with HG group, $P<0.05$.

Abbreviations: AS-IV, Astragaloside IV; HG, high glucose; MC, mesangial cell.

after high glucose exposure for 1 hour, and then with AS-IV for 48 hours. While SRT1720 significantly increased Beclin 1 expression, AS-IV and EX527 co-treatment decreased Beclin 1 levels in the MCs (Figure 4A). Similarly, LC3 II level was significantly increased in MCs pro-treated with SRT1720, but decreased in MCs co-treated with EX527 and AS-IV (Figure 4B). In addition, both autophagy markers were increased in the presence of SRT1720 and were inhibited by EX527 in MCs under hyperglycemic condition, independent of AS-IV (Figure 4A and B). Therefore, AS-IV promoted autophagy in hyperglycemia-activated MCs by promoting SIRT1-mediated deacetylation of NF- $\kappa$ B p65 subunit.
We also detected the levels of the autophagy markers in renal tissues and found them significantly decreased in the untreated DKD mice kidneys compared to the NC mice, and increased in the AS-IV-treated DKD mice (Figure 4C and D).

\section{Effect of AS-IV on hyperglycemia-inhibited autophagy to suppress MCs proliferation}

Autophagy maintains intracellular homeostasis by degrading damaged cellular components. To further explore the role of autophagy in hyperglycemia-induced MC proliferation, the cells were treated with the autophagy inhibitor 3-MA or 


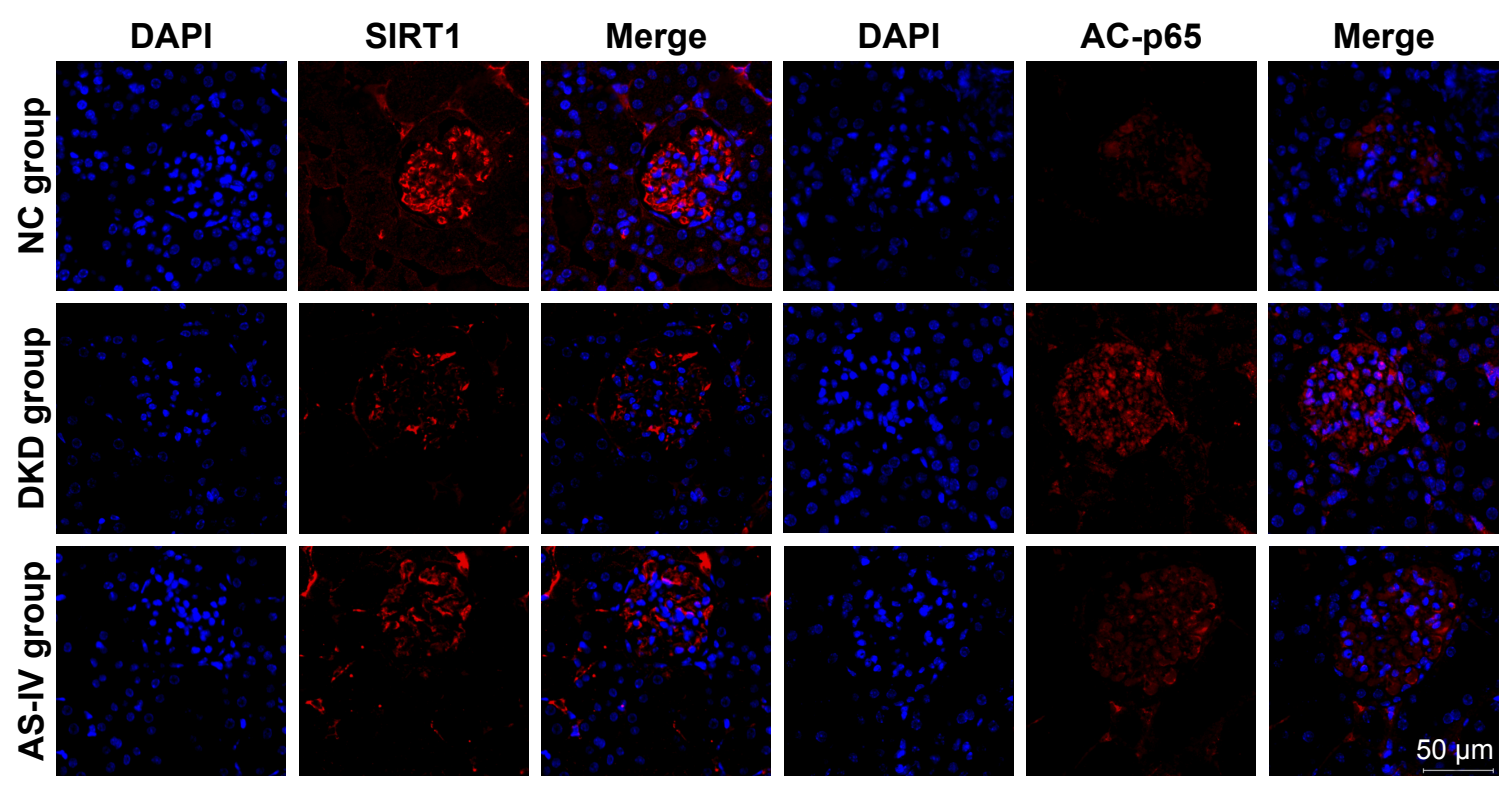

Figure 3 Effect of AS-IV on SIRTI and NF-KB p65 expression in vivo.

Notes: Immunofluorescence images showed that the expression of SIRTI was decreased and p65 acetylation was increased in DKD group mice glomerulus compared with NC group mice glomerulus and that AS-IV treatment increased SIRTI expression and decreased p65 acetylation expression in glomerulus.

Abbreviations: AS-IV, Astragaloside IV; DKD, diabetic kidney disease; NC, normal control.

activator rapamycin after high glucose exposure, followed by AS-IV treatment for 48 hours. AS-IV decreased the proliferation rate of MCs and this ameliorative effect was inhibited in the presence of 3-MA (Figure 5A), while rapamycin suppressed MC proliferation. Taken together, AS-IV inhibited hyperglycemia induced $\mathrm{MC}$ proliferation by enhancing autophagy (Figure 5A).

A
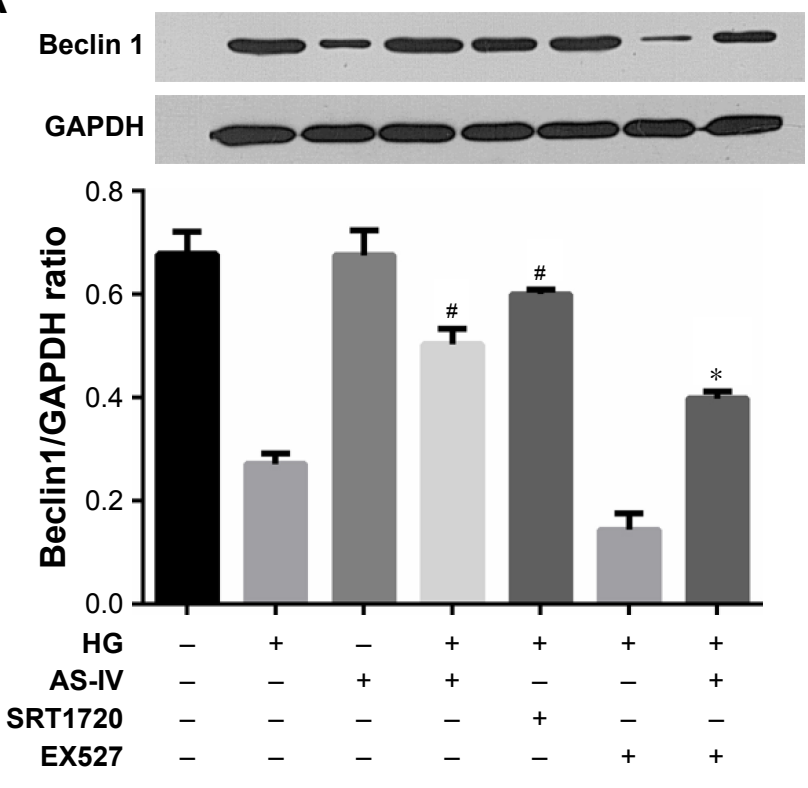

Figure 4 (Continued)

\section{Effect of AS-IV-induced autophagy on hyperglycemia-activated MCs}

To explore the effect of AS-IV induced autophagy on hyperglycemia mediated MC activation, the cells were exposed to high glucose levels for 1 hour and then treated with 3-MA for another hour, and incubated in the presence of AS-IV for 48 hours. AS-IV and hyperglycemia led to a greater decrease

B
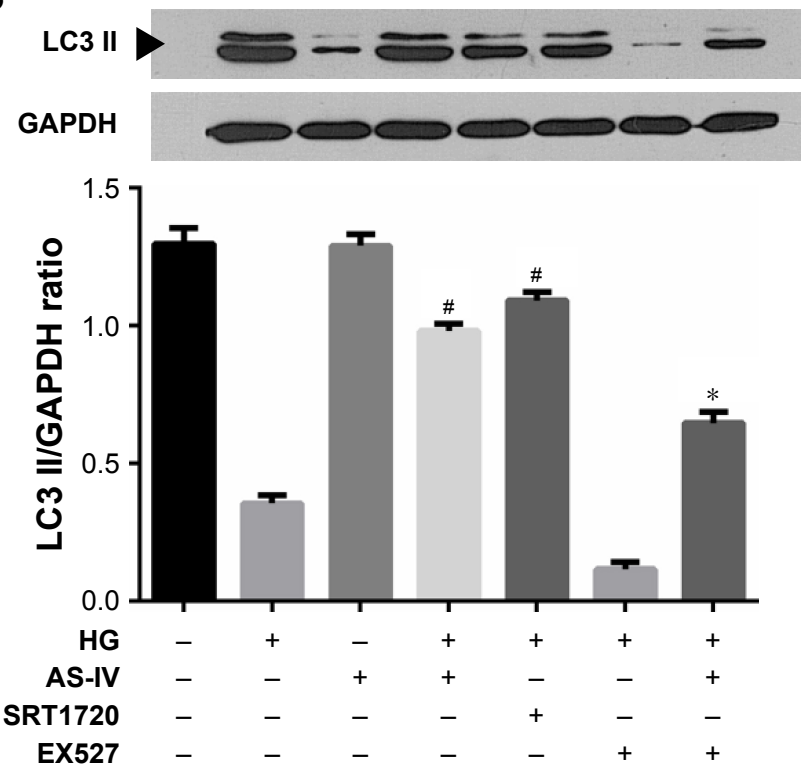


\section{C}
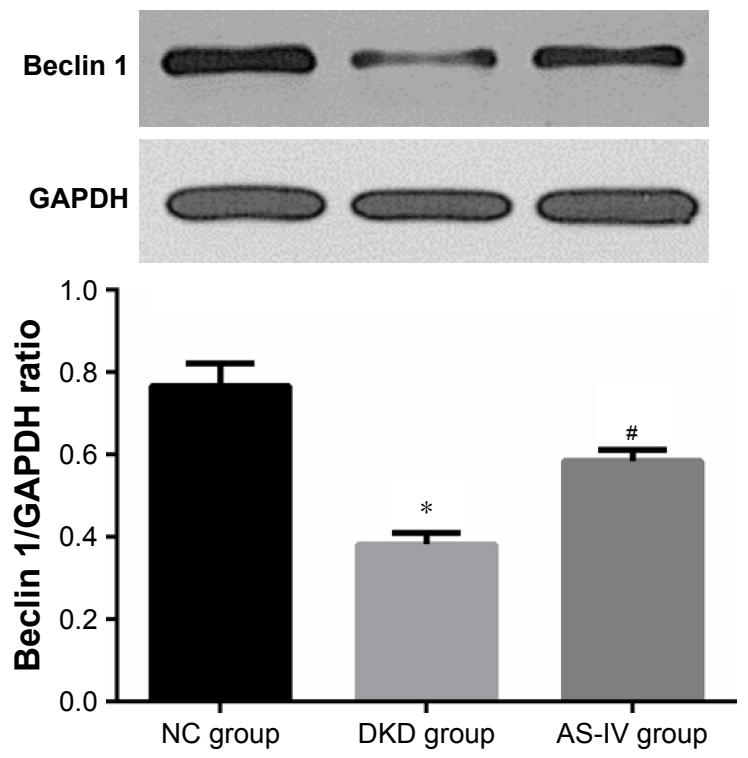

D

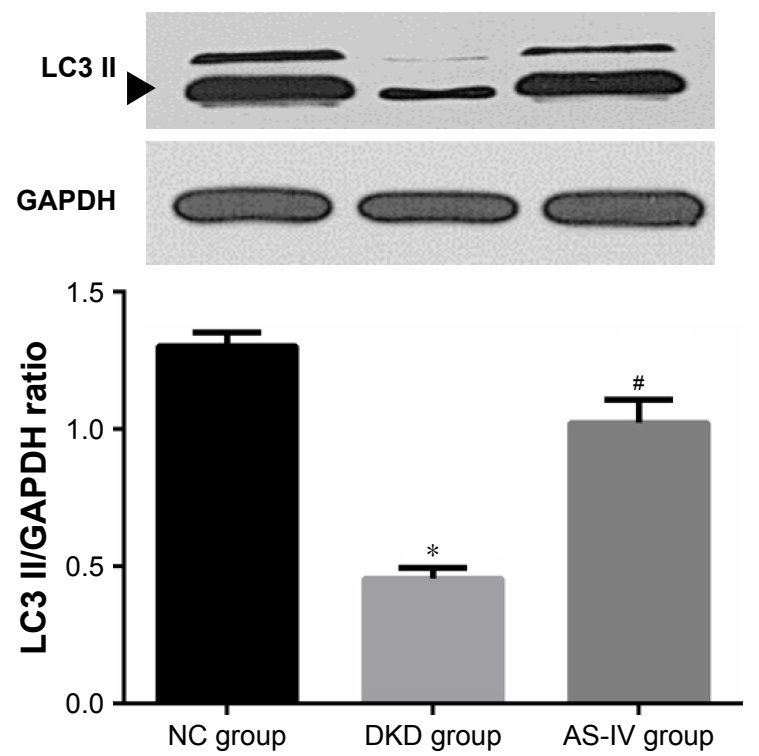

Figure 4 Effect of AS-IV on SIRTI-induced inactivation of NF-KB signaling results in autophagy in hyperglycemia-induced MCs activation.

Notes: (A and B) Real-time PCR and Western blotting results showed that both SIRTI activator SRTI720 and AS-IV increased the level of autophagy markers (Beclin I and LC3 II) in MCs exposed to hyperglycemia for 48 hours, and that SIRTI inhibitor EX527 abolished the change of AS-IV. Data are presented as mean \pm SD; $\mathrm{n}=3$. *Compared with co-treated with hyperglycemia and AS-IV group, $P<0.05$. " Compared with HG group, $P<0.05$. (C and $\mathbf{D})$ Western blotting results showed that AS-IV treatment increased the expression of Beclin I and LC3 II in renal tissues compared to the untreated DKD mice. Data are presented as mean \pm SD; $\mathrm{n}=3$. $*$ Compared with NC group, $P<0.05$. "Compared with DKD group, $P<0.05$.

Abbreviations: AS-IV, Astragaloside IV; DKD, diabetic kidney disease; HG, high glucose; MC, mesangial cell; NC, normal control.

A

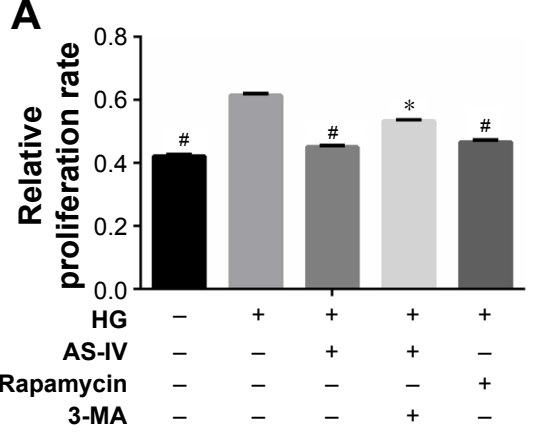

D

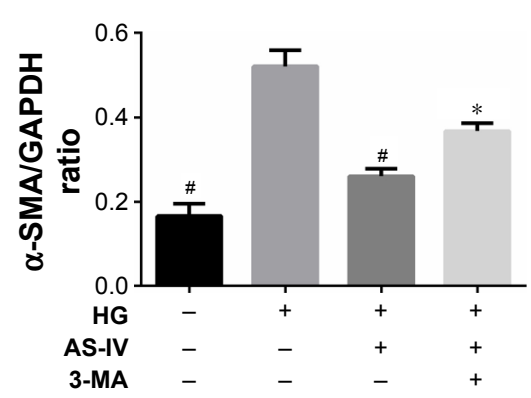

B

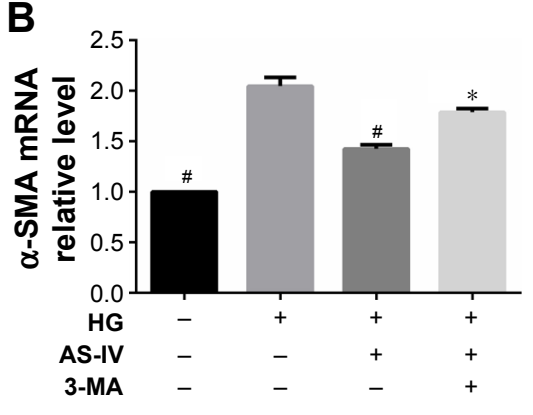

E

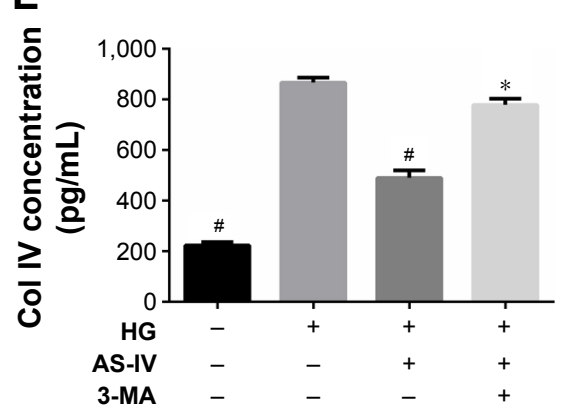

C

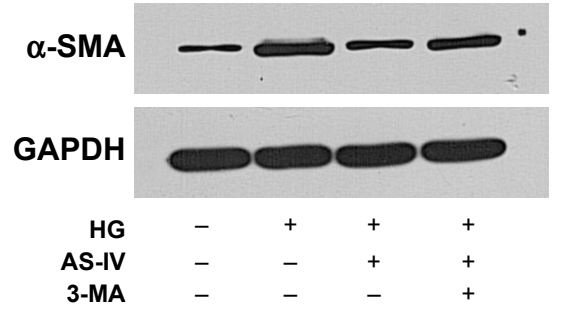

F

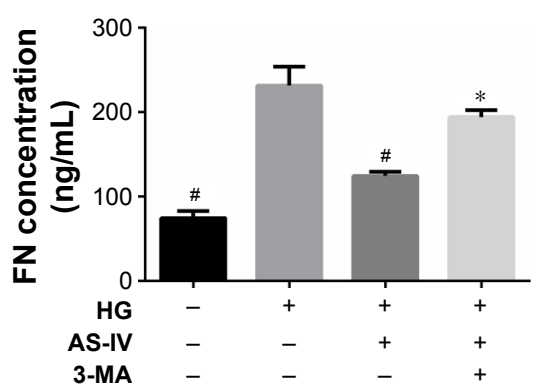

Figure 5 Effect of AS-IV-induced autophagy on hyperglycemia-activated MCs.

Notes: (A) CCK-8 assay results showed that both autophagy activator rapamycin and AS-IV (I00 $\mu$ M) decreased the proliferation rate in MCs exposed to hyperglycemia for 48 hours and that autophagy inhibitor 3-MA abolished the change of AS-IV. (B-D) Real-time PCR and Western blotting results showed AS-IV (I00 $\mu$ M) decreased the relative $\alpha$-SMA levels in MCs exposed to hyperglycemia for 48 hours and that autophagy 3-MA abolished this effect. (E and F) ELISA assay results showed AS-IV (I00 $\mu$ M) decreased the levels of FN and Col IV in hyperglycemia-stimulated MCs for 48 hours and that 3-MA reversed this change. Data are presented as mean \pm SD; $\mathrm{n}=3$. *Compared with co-treated with hyperglycemia and AS-IV group, $P<0.05$. ${ }^{*}$ Compared with HG group, $P<0.05$.

Abbreviations: AS-IV, Astragaloside IV; HG, high glucose; MC, mesangial cell. 

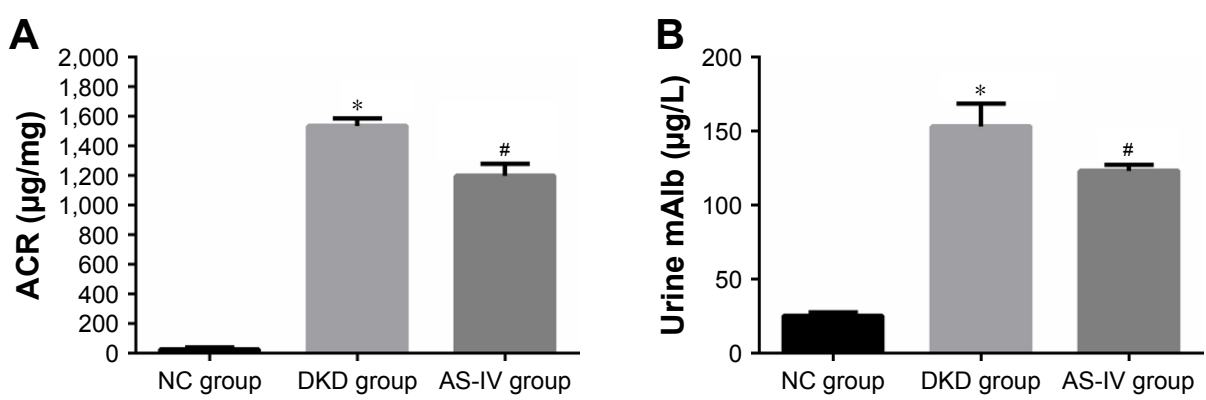

C

NC group
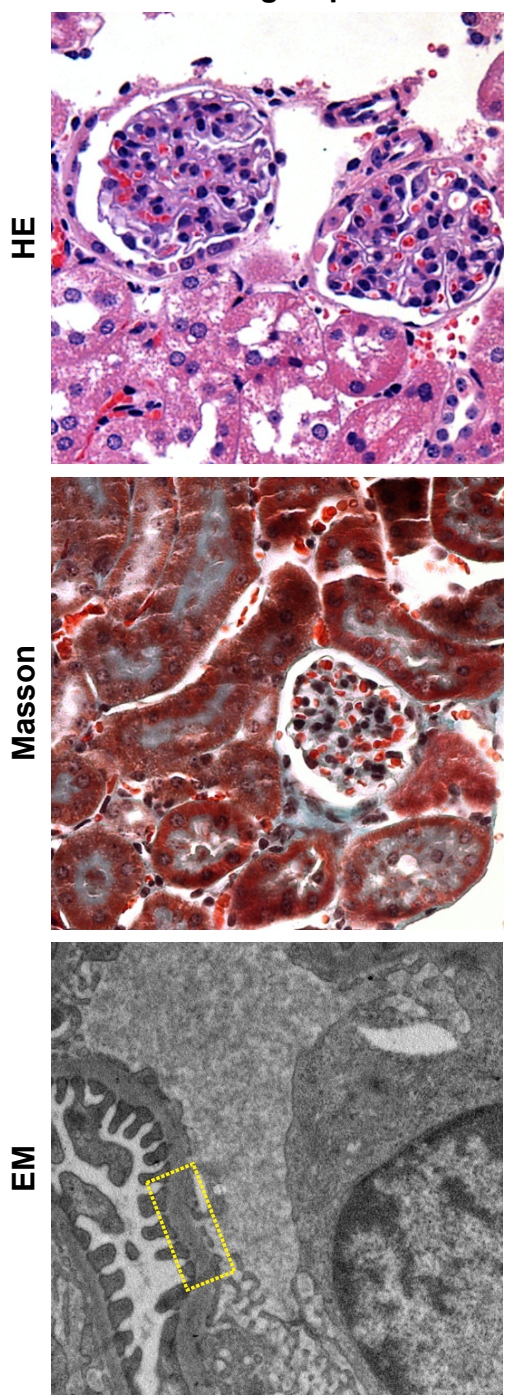

DKD group

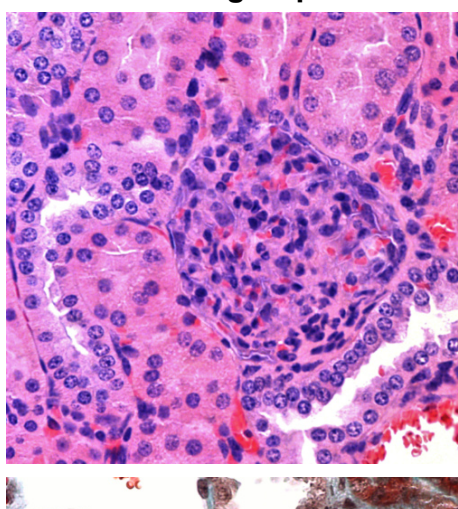

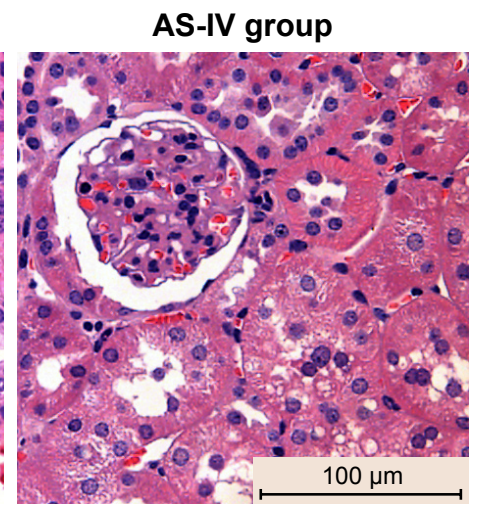
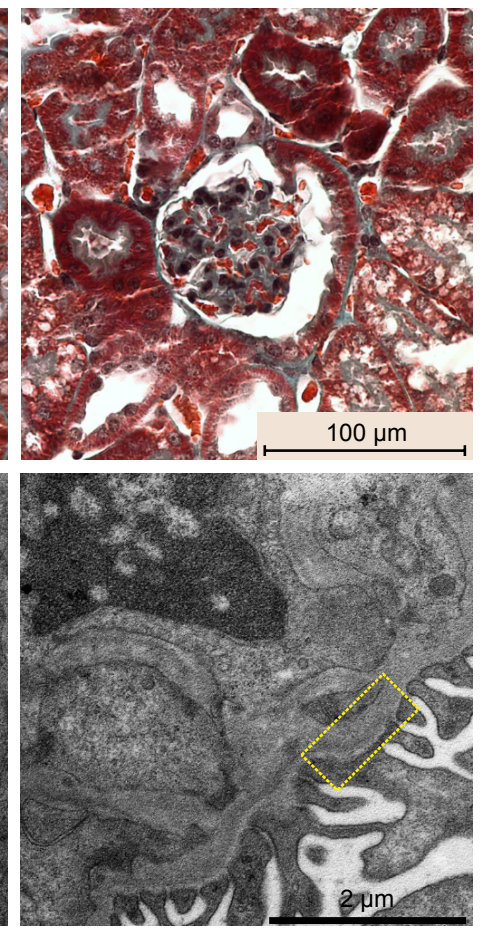

Figure 6 Effect of AS-IV on renal function and renal morphology in diabetic KK-Ay mice.

Notes: (A and B) The data showed AS-IV treatment decreased ACR and mAlb compared to the untreated DKD mice. (C) Light and electron microscopy images showed that AS-IV treatment decreased the thickness of GBM and reduced mesangial expansion, proliferation, and ECM protein overproduction compared to the untreated DKD mice. Data are presented as mean $\pm \mathrm{SD} ; \mathrm{n}=3$. ${ }^{*}$ Compared with NC group, $P<0.05$. ${ }^{*}$ Compared with DKD group, $P<0.05$. The dotted yellow boxes indicate the GBM.

Abbreviations: ACR, albumin creatinine ratio; AS-IV, Astragaloside IV; DKD, diabetic kidney disease; ECM, extracellular matrix; EM, electron microscopy; GBM, glomerular basement membrane; HE, hematoxylin-eosin; mAlb, microalbuminuria; NC, normal control.

in $\alpha$-SMA mRNA and protein levels in MCs compared to hyperglycemia alone, and this effect was reversed in the presence of 3-MA (Figure 5B-D). Similarly, AS-IV-mediated decrease in $\mathrm{FN}$ and $\mathrm{Col}$ IV levels was also abolished by 3-MA during hyperglycemia-induced $\mathrm{MC}$ activation (Figure 5E and F). These results strongly suggested that AS-IV impeded the MC activation induced by hyperglycemia by enhancing autophagy. 


\section{Effect of AS-IV on renal function and morphology in diabetic KK-Ay mice}

To explore the effect of AS-IV on renal function and morphology in KK-Ay mice, ACR and microalbuminuria (mAlb) levels were measured in the urine, and renal tissues were analyzed by routine hematoxylin-eosin and Masson staining. Furthermore, electron microscopy (EM) was used to observe the thickness of glomerular basement membrane (GBM). AS-IV treatment decreased urine ACR and mAlb levels (Figure 6A and B) and also alleviated mesangial expansion, proliferation, and ECM protein overproduction compared to the untreated DKD mice (Figure 6C). In addition, AS-IV treated mice had thinner GBM and better glomerular foot process fusion compared to the untreated DKD mice (Figure 6C). Taken together, AS-IV improved renal function and morphology in diabetic KK-Ay mice.

\section{Discussion}

$\mathrm{MC}$ activation plays a vital role in the progression of glomerulosclerosis in DKD. ${ }^{22}$ It can be triggered by various pathological conditions such as oxidative stress and autophagy. Several studies have shown the effect of AS-IV on autophagy in various cells, ${ }^{18,23,24}$ including the MCs. We examined the effect of autophagy on MC activation and found that hyperglycemia activated the MCs and decreased the expression of LC3-II and Beclin 1. AS-IV treatment reversed these changes, suggesting that AS-IV could enhance autophagy during hyperglycemia-induced MC activation.

Several studies have shown a crucial role of SIRT1 in regulating $\mathrm{MC}$ injury. ${ }^{9,10,25}$ For instance, activation of SIRT1 retarded the ECM accumulation in MCs exposed to TGF- $\beta 1 .{ }^{25}$ There are also reports of a direct effect of SIRT1 on autophagy regulation ${ }^{26-28}$ through the deacetylation of autophagy-related proteins such as Atg5 and Atg $7 .{ }^{26}$ Chuang et $\mathrm{a}^{28}$ showed that podocyte autophagy was suppressed in SIRT1 knockout mice. However, only a few studies have reported on the relationship of SIRT1 and autophagy in MC injury. To elucidate this point, we tested the respective effects of an SIRT1 activator and an inhibitor on autophagy in hyperglycemia-activated MCs. LC3-II and Beclin 1 levels were significantly increased in MCs treated with SRT1720, and decreased after EX527 treatment. These findings indicate that SIRT1 activates autophagy in hyperglycemiaactivated MCs.

Autophagy can also be modulated by NF-kB signaling. ${ }^{29-31}$ Wei et $\mathrm{al}^{30}$ reported that activation of NF- $\mathrm{KB}$ signaling suppressed autophagy in hyperglycemia-induced podocytes by downregulating LC3-II. In this study, we found that hyperglycemia suppressed autophagy in MCs along with activating NF- $\mathrm{KB}$ signaling, both of which were reversed by AS-IV treatment. The possible mechanism employed by AS-IV is the inhibition of NF-kB signaling by enhancing SIRT1 deacetylation. To prove this hypothesis, MCs were pretreated with the SIRT1 activator or inhibitor. As seen with AS-IV treatment, MCs treated with SRT1720 showed enhanced autophagy and lower NF- $\kappa$ B p65 acetylation levels, while a completely opposite effect was seen with EX527 treatment. Therefore, AS-IV acts as an SIRT1 activator to inhibit the acetylation of NF- $\mathrm{\kappa B}$ p 65 subunit and enhances autophagy in hyperglycemia-activated MCs. Furthermore, both AS-IV and rapamycin (autophagy activator) suppressed hyperglycemia-induced MC proliferation, while co-treatment with AS-IV and 3-MA (autophagy inhibitor) promoted MC proliferation. These findings suggest that AS-IV-mediated autophagy protects against MC injury under hyperglycemic conditions. Furthermore, our results showed that AS-IV impeded MC activation, which was reversed by 3-MA. Taken together, AS-IV inhibits hyperglycemia-induced MC activation by enhancing autophagy through the SIRT1NF- $\kappa B$ pathway. However, 3-MA could not fully inhibit the restorative effects of AS-IV, indicating the involvement of other signaling mechanisms.

In summary, our study suggests that AS-IV ameliorates renal function and morphology in diabetic KK-Ay mice and inhibits hyperglycemia-induced $\mathrm{MC}$ activation by enhancing autophagy and inhibiting NF- $\mathrm{\kappa B}$ signaling by SIRT1 deacetylation. These findings provide the experimental basis to further investigate the therapeutic effects of AS-IV on glomerular diseases.

\section{Acknowledgment}

This study was supported by a grant from the Major National Basic Research Program of China (973 Program, no 2012CB518602).

\section{Disclosure}

The authors report no conflicts of interest in this work.

\section{References}

1. Dronavalli S, Duka I, Bakris GL. The pathogenesis of diabetic nephropathy. Nat Clin Pract Endocrinol Metab. 2008;4(8):444-452.

2. Liu HF, Liu H, Lv LL, et al. CCN3 suppresses TGF- $\beta 1$-induced extracellular matrix accumulation in human mesangial cells in vitro. Acta Pharmacol Sin. 2018;39(2):222-229.

3. Abboud HE. Mesangial cell biology. Exp Cell Res. 2012;318(9): 979-985.

4. Chuang PY, Cai W, Li X, et al. Reduction in podocyte SIRT1 accelerates kidney injury in aging mice. Am J Physiol Renal Physiol. 2017;313(3) F621-F628. 
5. Nakatani Y, Inagi R. Epigenetic Regulation Through SIRT1 in Podocytes. Curr Hypertens Rev. 2016;12(2):89-94.

6. Chang HC, Guarente L. SIRT1 mediates central circadian control in the SCN by a mechanism that decays with aging. Cell. 2013;153(7): $1448-1460$

7. Wakino S, Hasegawa K, Itoh H. Sirtuin and metabolic kidney disease. Kidney Int. 2015;88(4):691-698.

8. Hariharan N, Maejima Y, Nakae J, Paik J, Depinho RA, Sadoshima J. Deacetylation of FoxO by Sirt1 plays an essential role in mediating starvation-induced autophagy in cardiac myocytes. Circ Res. 2010; 107(12):1470-1482.

9. Shang G, Gao P, Zhao Z, et al. 3,5-Diiodo-1-thyronine ameliorates diabetic nephropathy in streptozotocin-induced diabetic rats. Biochim Biophys Acta. 2013;1832(5):674-684.

10. Kume S, Haneda M, Kanasaki K, et al. SIRT1 inhibits transforming growth factor beta-induced apoptosis in glomerular mesangial cells via Smad7 deacetylation. J Biol Chem. 2007;282(1):151-158.

11. Cybulsky AV. Endoplasmic reticulum stress, the unfolded protein response and autophagy in kidney diseases. Nat Rev Nephrol. 2017;13(11):681-696.

12. Xu L, Fan Q, Wang X, Zhao X, Wang L. Inhibition of autophagy increased AGE/ROS-mediated apoptosis in mesangial cells. Cell Death Dis. 2016;7(11):e2445.

13. Lu X, Fan Q, Xu L, et al. Ursolic acid attenuates diabetic mesangial cell injury through the up-regulation of autophagy via miRNA-21/PTEN/ Akt/mTOR suppression. PLoS One. 2015;10(2):e0117400.

14. Fujishiro H, Liu Y, Ahmadi B, Templeton DM. Protective effect of cadmium-induced autophagy in rat renal mesangial cells. Arch Toxicol. 2018;92(2):619-631.

15. Cai Z, Liu J, Bian H, Cai J. Astragaloside IV ameliorates necrotizing enterocolitis by attenuating oxidative stress and suppressing inflammation via the vitamin $\mathrm{D} 3$-upregulated protein $1 / \mathrm{NF}-\mathrm{\kappa B}$ signaling pathway. Exp Ther Med. 2016;12(4):2702-2708.

16. Sun L, Li W, Li W, Xiong L, Li G, Ma R. Astragaloside IV prevents damage to human mesangial cells through the inhibition of the NADPH oxidase/ROS/Akt/NF- $\mathrm{\kappa B}$ pathway under high glucose conditions. Int $J$ Mol Med. 2014;34(1):167-176.

17. Chen X, Wang DD, Wei T, He SM, Zhang GY, Wei QL. Effects of astragalosides from Radix Astragali on high glucose-induced proliferation and extracellular matrix accumulation in glomerular mesangial cells. Exp Ther Med. 2016;11(6):2561-2566.

18. Guo H, Wang Y, Zhang X, et al. Astragaloside IV protects against podocyte injury via SERCA2-dependent ER stress reduction and AMPK $\alpha$-regulated autophagy induction in streptozotocin-induced diabetic nephropathy. Sci Rep. 2017;7(1):6852.
19. Schmittgen TD, Lee EJ, Jiang J, et al. Real-time PCR quantification of precursor and mature microRNA. Methods. 2008;44(1):31-38.

20. Pfaffl MW. A new mathematical model for relative quantification in real-time RT-PCR. Nucleic Acids Res. 2001;29(9):e45.

21. Nopparat C, Sinjanakhom P, Govitrapong P. Melatonin reverses $\mathrm{H}_{2} \mathrm{O}_{2}$ induced senescence in SH-SY5Y cells byenhancing autophagy via

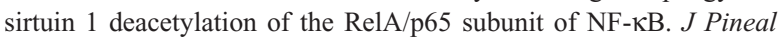
Res. 2017;63(1):e12407.

22. Shemesh II, Rozen-Zvi B, Kalechman Y, Gafter U, Sredni B. AS101 Prevents Diabetic Nephropathy Progression and Mesangial Cell Dysfunction: Regulation of the AKT Downstream Pathway. PLoS One. 2013;9(12):e114287.

23. Dong Z, Zhou J, Zhang Y, et al. Astragaloside-IV Alleviates HeatInduced Inflammation by Inhibiting Endoplasmic Reticulum Stress and Autophagy. Cell Physiol Biochem. 2017;42(2):824-837.

24. Liu J, Meng Q, Jing H, Zhou S. Astragaloside IV protects against apoptosis in human degenerative chondrocytes through autophagy activation. Mol Med Rep. 2017;16(3):3269-3275.

25. Huang XZ, Wen D, Zhang M, et al. Sirt1 activation ameliorates renal fibrosis by inhibiting the TGF- $\beta / \operatorname{Smad} 3$ pathway. $J$ Cell Biochem. 2014;115(5):996-1005.

26. Lee IH, Cao L, Mostoslavsky R, et al. A role for the NAD-dependent deacetylase Sirt1 in the regulation of autophagy. Proc Natl Acad Sci US A. 2008;105(9):3374-3379.

27. Huang R, Xu Y, Wan W, et al. Deacetylation of nuclear LC3 drives autophagy initiation under starvation. Mol Cell. 2015;57(3):456-466.

28. Chuang PY, Xu J, Dai Y, et al. In vivo RNA interference models of inducible and reversible Sirt1 knockdown in kidney cells. Am J Pathol. 2014;184(7):1940-1956.

29. Hu W, Chen SS, Zhang JL, Lou XE, Zhou HJ. Dihydroartemisinin induces autophagy by suppressing NF- $\mathrm{\kappa B}$ activation. Cancer Lett. 2014;343(2):239-248.

30. Wei M, Li Z, Yang Z. Crosstalk between protective autophagy and NF- $\mathrm{KB}$ signal in high glucose-induced podocytes. Mol Cell Biochem. 2014;394(1-2):261-273.

31. Lin F, Ghislat G, Luo S, Renna M, Siddiqi F, Rubinsztein DC. XIAP and cIAP1 amplifications induce Beclin 1-dependent autophagy through NFKB activation. Hum Mol Genet. 2015;24(10):2899-2913.
Drug Design, Development and Therapy

\section{Publish your work in this journal}

Drug Design, Development and Therapy is an international, peerreviewed open-access journal that spans the spectrum of drug design and development through to clinical applications. Clinical outcomes, patient safety, and programs for the development and effective, safe, and sustained use of medicines are the features of the journal, which

\section{Dovepress}

has also been accepted for indexing on PubMed Central. The manuscript management system is completely online and includes a very quick and fair peer-review system, which is all easy to use. Visit http://www.dovepress.com/testimonials.php to read real quotes from published authors. 